\title{
LES THÉORIES DE LA COMMUNICATION COMME MÉTAPHORES QUI SE RÉALISENT
}

\author{
Jean-Pierre Meunier ${ }^{1}$
}

\section{Métaphores théoriques}

Toutes sortes d'images hantent l'élaboration des théories scientifiques, images d'origine souvent technicienne qui impriment leurs formes sur les structures conceptuelles construites par les chercheurs. $\mathrm{Au} \mathrm{XIX}^{\mathrm{e}}$ siècle, à l'époque de la machine à vapeur et de la thermodynamique, Freud conçoit son appareil psychique comme une structure compartimentée traversée par des courants énergétiques (la libido, les pulsions), tandis que Marx fait reposer la machine sociale sur les forces productives. Influences accidentelles liées à une percée spectaculaire de la science et de la technique? Il ne semble pas. Un coup d'oeil sur l'histoire montre la constance de l'influence de la technique sur l'imaginaire scientifique. "Depuis le XVII" siècle, note Pierre Lévy, notre notion de causalité se meut dans l'univers des chocs, des poussées et des engrenages, du mécanisme, etc. On n'en finirait pas d'énumérer les emprunts de la pensée dite abstraite (en fait métaphorique) aux modèles techniques les plus quotidiens. Non seulement les concepts sont nomades, passent d'un territoire du savoir à l'autre, mais ils sont presque toujours aussi de basse extraction, fils de paysans,

1 Professeur à l'Université catholique de Louvain. Président du Département de communication.

Recherches en communication, $\mathrm{n}^{\circ} 1$ (1994). 
d'artisans, de techniciens, de travailleurs manuels"1. Notre époque, marquée par l'apparition des machines informationnelles autant que le $\mathrm{XIX}^{\mathrm{e}}$ l'avait été par les machines énergétiques, voit se former dans l'imaginaire des sciences humaines des notions et des représentations de fonctionnement directement inspirées par les nouvelles machines: programme, computation, traitement d'information ... On y reviendra plus loin.

Les théories qui, depuis quelques dizaines d'années, tentent de formaliser les processus à l'oeuvre dans la communication dépendent évidemment de telles migrations métaphoriques. Toutes sortes d'images ont guidé l'imagination des chercheurs en communication: celle du télégraphe avec ses lignes de transmission et son alphabet morse, celle du jeu d'échec avec ses stratégies et ses "coups", celle de l'orchestre, ou, tout récemment, celle du traitement informatique de l'information. Que dire alors des modèles théoriques produits par de telles images? Ne seraient-ils que pures constructions mentales, subjectives, imaginaires, inessentielles, contingentes, et pour tout dire sans grand rapport avec la réalité des choses? On perçoit l'ampleur du débat et la complexité des problèmes à résoudre: qu'est-ce qu'une métaphore, de quelle manière intervient-elle dans notre représentation des choses, quel rapport entre celle-ci et le réel?

Admettons pour commencer cette définition de la métaphore formulée par Lakoff et Johnson: "L'essence d'une métaphore est qu'elle permet de comprendre quelque chose (et d'en faire l'expérience) en termes de quelque chose d'autre"2. Cette définition, on le voit, s'éloigne assez des figures de style de la rhétorique; elle concerne la compréhension, c'est-à-dire la connaissance conceptuelle du "réel". Elle n'implique pas que cette connaissance soit purement illusoire mais que, procédant souvent -si pas toujours- par analogie, elle soit forcément partielle, ne percevant des phénomènes que les aspects que font précisément ressortir les métaphores, et ignorant les autres aspects. Mais, plus important, la métaphore selon Lakoff et Johnson ne concerne pas la seule connaissance -comme si celle-ci se tenait toujours à l'écart du réel, n'ayant pour but que de le re-présenter sans le toucher. Elle a aussi quelque chose à voir avec notre expérience des choses et notre action.

1 Pierre LÉVy, Les technologies de l'intelligence, Paris, La Découverte, 1990, p. 79.

2 George LAKoff et Mark JoHnson, Les métaphores dans la vie quotidienne, (trad. fr.), Paris, Éd. de Minuit, 1983, p. 15. 
"Les métaphores peuvent créer des réalités, en particulier des réalités sociales. Une métaphore peut être un guide pour l'action future. Les actions futures s'ajusteront à la métaphore. En retour, le pouvoir qu'a la métaphore de rendre cohérente l'expérience en sera renforcé. En ce sens, les métaphores peuvent être des prophéties qui engendrent leur propre accomplissement"1.

Les métaphores ont donc quelque chose des prophéties autoréalisatrices. C'est cet aspect de leur fonctionnement que l'on voudrait mettre ici en évidence à propos des théories de la communication. Précisons tout d'abord cette notion de prophétie autoréalisatrice. Dans l'épistémologie courante, le réel et sa représentation sont sans influence réciproque. Le réel est là, avant tout regard que l'on peut poser sur lui et la représentation n'a précisément à charge que de la représenter. Cette conception commande nos idées sur la vérité de nos énoncés (scientifiques, journalistiques, ...): sera considéré comme vrai ou objectif ce qui semblera correspondre à la réalité. A l'opposé de cette conception, on voit se dessiner dans l'épistémologie contemporaine une tendance à considérer nos représentations plutôt comme des constructions de la réalité. Pour le "constructivisme" par exemple, la connaissance est moins le reflet du réel que la mise en ordre, l'organisation du monde par notre intelligence 2 .

Dans cette perspective, on comprend l'importance du rôle cognitif de la métaphore: en nous faisant voir des choses dans les termes d'autres choses, elle intervient en effet très activement dans l'organisation de notre expérience du monde.

Mais il faut concevoir le constructivisme comme dépassant le cadre de la connaissance. Dans la mesure où la connaissance commande l'action, elle intervient forcément aussi dans la construction -en un sens plus fort du terme- de la réalité, tout au moins de la réalité sociale. C'est dans cette perspective que prend sens la notion de prophétie autoréalisatrice. Il faut concevoir une relation récursive comprenant différentes étapes que l'on pourrait caractériser de la manière suivante:

1 Ibidem, p. 166.

2 Cf. à ce sujet P. Watzlawick (sous la dir. de), L'invention de la réalité, contributions au constructivisme, Paris, Éd. du Seuil, 1988. 
1. Dans les interactions liant les hommes entre eux et avec le monde, surgissent des représentations -des hommes eux-mêmes, de leurs rapports, du monde-.

2. Ces représentations soit restent marginales, soit prennent de l'importance et se généralisent par un processus d'amplification ou intervient certainement le conformisme, ou mieux le mimétisme, qui pousse les hommes au partage des mêmes idées, valeurs, conduites, ...

3. En même temps, les individus agissant de plus en plus en fonction de ces représentations, génèrent une réalité qui leur devient conforme.

4. Dans la nouvelle réalité surgissent de nouvelles représentations, etc.

J.-P. Dupuy, qui a consacré quelques belles pages à la prophétie autoréalisatrice - en la situant dans le cadre de la théorie des systèmes autoréférentiels- précise : "Un équilibre, c'est-à-dire un point fixe du processus autoréférentiel, est obtenu lorsque les réactions des acteurs aux représentations qu'ils se font de la réalité dans laquelle ils se trouvent plongés engendrent par effet de composition une réalité conforme à ces représentations". ${ }^{1}$

Parlons plus concrètement. Dans notre culture, l'amour est généralement -selon certaines métaphores devenues conventionnellesperçu comme un voyage ou comme une folie, etc. Mais supposons comme l'imaginent Lakoff et Johnson, que s'impose cette métaphore nouvelle: l'amour est une œuvre d'art réalisée en commun. Il ne fait pas de doute qu'une telle métaphore ne donne une signification nouvelle à notre expérience de l'amour; il ne fait pas de doute non plus qu'elle ne transforme nos actions ${ }^{2}$.

"Beaucoup de nos activités (discuter, résoudre des problèmes,
gérer son temps, etc.) sont de nature métaphorique. Les
concepts métaphoriques qui caractérisent ces activités structu-
rent notre réalité actuelle. Mais les métaphores nouvelles ont
le pouvoir de créer une nouvelle réalité. Celle-ci peut
apparaître quand nous commençons à comprendre notre
expérience en termes d'une métaphore nouvelle et elle prend
de la distance quand nous commençons à agir en termes de
cette métaphore. Si une nouvelle métaphore entre dans le
système conceptuel sur lequel nous fondons nos actions, elle le

1 Jean-Pierre Dupuy, Ordres et désordres, Paris, Éd. du Seuil, 1982, p. 149.

2 George LAKOFF et Mark JoHnSON, op. cit., p. 149 et sv. 
modifiera ainsi que les perceptions que le système engendre. Bien des changements culturels ont pour cause l'introduction de nouveaux concepts métaphoriques et la perte d'anciens"'.

Cependant, la culture a ses inerties. Pour qu'une représentation nouvelle -métaphorique ou autre-engendre un véritable changement culturel, il faut ces processus de généralisation et d'amplification par renforcement mutuel dont nous parlions dans notre point 1 .

Nous pouvons maintenant mieux préciser notre point de vue au sujet des métaphores théoriques, plus particulièrement des théories (métaphoriques) de la communication. D'après ce qui vient d'être dit, on doit concevoir ces théories moins comme des tentatives plus ou moins réussies de représentation de processus leur restant de toute façon extérieurs que comme des moments de la boucle de la prophétie autoréalisatrice dont on vient de décrire les étapes essentielles. Nous allons examiner de ce point de vue les modèles théoriques qui ont marqué l'histoire, toute récente encore, des recherches en matière de communication.

\section{Le modèle du code et la métaphore du télégraphe}

On sait l'importance qu'a prise, dans l'ensemble des recherches s'occupant de communication, le modèle théorique proposé par Shannon et Weaver. Les éléments essentiels de ce modèle sont l'émetteur (qui envoie l'information), le récepteur (qui la reçoit), le message (qui circule de l'émetteur au récepteur), et le code (qui permet la traduction en signes des idées transmises par le message). Ce modèle élaboré par des ingénieurs de télécommunications (Shannon travaille pour la compagnie Bell Telephone) mais adopté ensuite par les sciences humaines, doit évidemment beaucoup à l'image du télégraphe, ainsi que l'a souligné avec force Y. Winkin: "Sans doute grâce à son extrême dépouillement, ce schéma est devenu le modèle de la communication en sciences sociales, tant aux États-Unis qu'en Europe. Certes, très nombreuses ont été les critiques et les modifications - mais on n'est pas sorti du couple émetteur-récepteur. Tout se passe comme si le seul élément que Shannon ait pu léguer aux non-

I Ibidem, p. 155. 
ingénieurs soit l'image du télégraphe (...). On pourrait ainsi parler d'un modèle télégraphique de la communication"'.

La sémiologie a généralisé ce modèle à l'ensemble des modes de communication. La pièce mâtresse du modèle étant le code, les sémiologues se sont donc ingéniés à mettre au jour les codes qui devraient régler -et rendre possible- la communication par l'image, le geste, le son, les objets, etc.

A vrai dire, la notion de code n'a pas attendu la théorie shannonnienne pour s'imposer à l'esprit des théoriciens. On peut, selon Sperber et Wilson trouver une telle notion chez Aristote, saint Augustin, les grammairiens de Port-Royal, etc. Ce qui montre des lointaines attaches avec nos habitudes de penser.

"L'idée selon laquelle la communication verbale est un processus de codage et de décodage est si profondément enracinée dans notre culture occidentale qu'on a tendance à oublier qu'il s'agit là, après tout, d'une hypothèse et pas d'un fait"'2.

Que l'idée selon laquelle la communication est un processus de codage/décodage soit profondément enracinée dans notre culture apparaît non seulement dans les écrits des théoriciens, mais aussi dans nos manières courantes de parler de la communication. Ces manières semblent inspirées par une métaphore qui en appelle à l'image du conduit. Ce sont des expressions comme "vous n'avez pas réussi à faire passer vos idées" qui font soupçonner cette imagerie. "Tout d'abord, la langue est vue comme un conduit qui transfère les pensées et les sentiments d'un individu à un autre. Ensuite, les expressions linguistiques, les mots, ce que Reddy appelle les signaux sont des contenants pour les concepts, les idées, appelés "membres du répertoire". Dans la communication elle-même, l'encodage s'effectue en "enveloppant" les idées dans les mots. Le décodage s'effectue lorsque l'auditeur/lecteur défait l'enveloppe des signaux pour en extraire les membres du répertoire et se les met dans l'esprit. Cette conception implique aussi que les idées sont éjectées dans un espace externe et sont réifiées dans cet espace" 3 .

Il ne fait pas de doute que toute cette imagerie ait quelque fondement, et même qu'elle révèle quelque aspect essentiel de la

1 Yves WinkIN, La nouvelle communication, Paris, Éd. du Seuil, 1981, p. 20.

2 Dan SPERBER et Deirdre WILSON, La pertinence, communication et cognition, Paris, Éd. de Minuit, 1989, p. 17.

3 A.-M. DilLER, "Cohérence métaphorique, action verbale et action mentale," in Communication, $\mathrm{n}^{\circ}$ 53, Paris, Éd. du Seuil, 1991, p. 221. 
communication en général. Après tout, on ne saurait parler de communication sans l'existence d'un minimum de séparation spatiale entre éléments communicants, sans aussi, quelque chose qui se transporte de l'un à l'autre et qui doit faire l'objet, à son point de départ comme à son point d'arrivée, d'un processus quelconque de transformation que l'on peut bien appeler encodage/décodage, à condition de donner à la notion de code un sens très général.

Mais il ne fait pas de doute non plus que cette imagerie occulte l'existence de divers autres aspects de la communication: celui sous lequel elle se présente comme une action par exemple -on y viendra plus loin-, l'activité de coopération qu'elle implique, la dimension empathique qui la soutient, etc.

La métaphore télégraphique n'a donc pas inventé une représentation vraiment nouvelle de la communication puisqu'on en trouve les éléments essentiels dans certaines théorisations anciennes ou dans les théories spontanées du sens commun. Mais elle a donné à cette représentation une force toute particulière. Dans les théories inspirées par Shannon, l'émetteur et le récepteur sont, psychologiquement et sociologiquement, aussi éloignés l'un de l'autre que le suggère l'image du télégraphe. L'émission et la réception sont des notions sans équivoque. Le code est une instance parfaitement autonome -socialement élaborée- et complètement indépendante des sujets communiquant. Les signes sont des entités tout à fait discrètes.

L'expression courante mentionnée plus haut ("il n'est pas arrivé à faire passer ses idées") repose pour l'essentiel sur l'image du conduit, mais elle réfère aussi implicitement au contact, à la confrontation, à la compréhension, etc. Dans les modèles shannonniens ou saussuriens, il n'est plus question que d'émission, encodage, transport, réception, décodage.

Si le modèle télégraphique s'est imposé avec autant de force dans les sciences humaines, c'est sans aucun doute parce qu'il fait droit à des aspects des processus concrets de communication qu'ont rendu saillants les technologies de communication inventées dans notre culture. L'écriture phonétique et l'imprimerie ont rendu évidentes, dans la représentation qu'on se fait de la communication, les entités qu'elles ont contribué à réaliser: les émetteurs et récepteurs comme entités séparées et la notion de code matérialisée dans les lexiques et dictionnaires. Les mass-media qui s'adressent forcément à des publics disséminés dans l'espace, ont littéralement concrétisé les notions d'émission et de réception. 
Mais le plus important, du point de vue adopté ici, est de montrer l'effet en retour du modèle télégraphique sur la culture. Dès l'instant où il impose son évidence scientifique, un modèle justifie et catalyse les pratiques qui s'y conforment. La définition scientifique -ou reconnue comme telle- induit forcément la norme. Il n'est de bonnes communications que celles qui répondent au modèle; les autres sont des communications ratées. Le modèle du code a généré toute une idéologie de la communication comme persuasion: une communication réussie est une communication dans laquelle un émetteur réussit à "faire passer" des choses (idées, images, croyances, affects, ...) dans la tête des gens. La plupart des spécialistes de la communication se sont identifiés à l'image du communicateur-stratège et leurs pratiques ont consisté pour l'essentiel à réaliser le modèle théorique au moyen d'un vaste encodage des choses échangées dans la communication.

\section{L'encodage de l'échange}

C'est ce processus d'encodage quasi forcené qu'ont saisi les sémiologues des années soixante. Encodage des vêtements -cf. le "système de la mode" de R. Barthes-, encodage des objets de consommation -cf. "le système des objets" décrit par J. Baudrillard-, encodage des événements dans les grandes catégories et formes de récit définies par les media d'information (fait divers, reportage en direct, face à face, cérémonies télévisées, etc., etc.). Nantis du modèle du code, les sémiologues ont dénoncé toutes ces formes d'encodage -pour Barthes, la sémiologie devait être envisagée comme une "sémioclastie"- mais il faut bien dire qu'en même temps, et forcément, ils participaient eux-mêmes au processus. L'absence d'alternative au modèle du code, la réitération des mêmes notions à travers toutes les analyses, ont verrouillé la représentation des processus et les processus eux-mêmes.

Il faut ici préciser un peu plus notre notion d'encodage des échanges sociaux. On le fera en référence à la publicité qui, parmi toutes les formes de communication typiques de notre culture, est la plus significative de l'évolution générale des communications. La réalisation du modèle du code repose sur une pratique globale décomposable en plusieurs opérations. Elle implique que l'on fasse des sujets auxquels on s'adresse de véritables "récepteurs", c'est-à-dire des êtres relativement incapables de prendre recul et de réagir aux messages 
qu'on leur propose. Le recours massif à l'image - du moins à un certain type d'image-contribue efficacement à cette transformation des sujets en récepteurs.

Par opposition au langage verbal dont l'usage implique une certaine prise de distance par rapport aux choses qu'il permet de désigner, l'image tend plutôt à exclure ce type de distanciation. L'image plonge le spectateur dans l'immédiateté : elle sollicite plus la participation affective et l'identification que la distanciation et la réflexion. C'est du reste, paradoxalement, ce qui fait son intérêt pédagogique. La participation affective constitue la base de la compréhension -au sens cognitif du terme- à condition toutefois que cette compréhension soit reprise et élaborée par la réflexion. Un bon film de fiction, un bon documentaire, un bon reportage se caractérisent par leur capacité à déclencher, au-delà des faits et événements qu'ils proposent à la saisie immédiate du spectateur, des prises de recul et des interrogations sur ces faits.

Mais les images publicitaires n'ont pas cette visée. Leur but est de maintenir, si l'on peut dire, le "récepteur" en état continuel de réception. Les images publicitaires -ou de propagande et, à leur suite, les images de la presse à sensation et même, dans une certaine mesure, de la presse sérieuse-- jouent de "l'accroche" -ce terme fait partie du vocabulaire technique des spécialistes de la communicationet de l'aveuglement. La mise en forme et le contenu des images, le jeu des couleurs et des formes, les gros plans qui maximalisent l'identification et collent, pour ainsi dire, les traits des personnages-modèles sont le visage des spectateurs, les caractères mêmes de ces modèles éclatant de beauté physique et de réussite, la répétition continuelle de telles images, tout cela n'a d'autre but que de maintenir constant l'état spectatoriel de "réception" en prévenant les prises de recul, toujours possibles. Les images -publicitaires, de propagande et, en général, des grands media- renvoient plus les unes aux autres, qu'elles ne réfèrent au monde réel. Leur but est moins d'ouvrir des perspectives sur le monde réel que d'enfermer le sujet dans les mondes imaginaires qu'elles posent à l'avant-plan du réel.

Les images, donc, transforment les sujets en "récepteurs". Mais cela ne suffit pas à réaliser le modèle du code. Celui-ci exige une autre opération, fondamentale, et qui mérite une attention toute particulière dans la mesure où, d'elle, dépend l'encodage proprement dit et donc la possibilité de faire en sorte que le sujet déjà transformé en récepteur devienne, en même temps un simple décodeur. Car cette 
possibilité ne va pas de soi. La mise en suspens, par l'image, des facultés de distanciation et de réflexion consciente ne supprime pas toute activité cognitive chez le récepteur. Au contraire, il apparâit qu'un certain niveau d'activité associative inconsciente s'en trouve libéré. Pour tel individu, une photographie montrant une manifestation de rue évoquera (selon une voie d'association par contiguilté) le contexte des difficultés économiques et sociales qui a suscité cette manifestation et la scène, dès lors, sera perçue et vécue en fonction de ce contexte: elle apparaîtra déprimante, ou justifiée, ou chargée de menace, ouvrant sur l'incertitude... Pour tel autre individu, la même scène photographiée évoquera (par analogie) toutes ces images de foules victorieuses produites par notre culture d'images (notamment cinématographiques) et sera par suite vécue comme une aventure exaltante. Le sujet-récepteur est en quelque sorte un sujet-rêveur, d'autant plus rêveur qu'il est coupé de l'échange discursif qui oblige les interlocuteurs à s'entendre sur le sens des termes. Son accommodation perceptive à l'image dépend largement des rapprochements qu'elle suscite en lui en fonction de sa propre expérience. L'encodage consiste en grande partie à orienter ou prédéterminer ces rapprochements par le moyen du montage (au sens large, englobant aussi bien le collage que le montage photographique ou cinématographique).

La pratique du montage (qui a connu différentes modalités techniques: collage au sens propre, photomontage, ...) suppose, du point de vue sémiologique, la constitution concomitante d'un répertoire d'éléments iconiques distincts. Ici aussi, il faut concevoir une circularité: la pratique du montage engendre la séparation des éléments qui soutient et active la pratique du montage. Les images du XIX ${ }^{\mathrm{e}}$ siècle (peintures, gravures, affiches, ...) donnaient surtout à voir des "Gestalt", c'est-à-dire des ensembles liés d'éléments saisis en contexte. Les images publicitaires se présentent plutôt comme des assemblages d'éléments préexistants. Elles relèvent d'une combinatoire, tout comme, du reste, les objets de consommation et personnages qu'elles ont à charge de faire valoir. C'est alors l'existence (quantitativement croissante) d'éléments iconiques distincts et combinables qui rend de plus en plus possible (et sûre) la construction de significations déterminées, simplement décodables par les sujets-récepteurs. Cette construction a pour principe d'agir sur les réseaux associatifs auxquels une image peut donner origine. Cette action peut s'exercer sur les voies d'association par contiguïté. Dans ce 
cas, il s'agira de faire figurer dans les "collages", des éléments qui, dans l'expérience commune, apparaissent indissociables de certains contextes et dont on peut attendre qu'ils infléchiront dans un sens donné la perception de l'ensemble. Par exemple, il suffira, comme dans une publicité pour la cigarette Kent, d'introduire dans l'image un élément habituellement vu dans les musées, un socle sur lequel on pose habituellement les objets d'art, pour y introduire en même temps l'atmosphère particulière à ce genre de lieu et y faire participer l'ensemble, dont le paquet de cigarettes. L'action sur les réseaux associatifs peut également emprunter la voie de la similarité. On retrouve ici la métaphore et ses effets cognitifs. Il s'agira en effet, en jouant sur la ressemblance, de faire percevoir un élément iconique en fonction d'un autre, déjà connu et expérimenté. Placé sur le socle de musée, le paquet de cigarettes Kent devient, du fait de sa position, semblable à un objet d'art et en revêt certains des attributs essentiels: beauté, pureté, sacralité.

Ainsi se constitue l'encodage des images: métonymiquement et métaphoriquement. Source d'interprétations multiples et d'ambiguïté, les associations par contiguité et/ou par similarité deviennent, lorsqu'elles sont ainsi orientées au moyen d'éléments bien choisis dans l'expérience culturelle commune, moyens de détermination du sens. A force de réitération, certaines images se trouvent assignées à l'évocation des domaines dont elles sont issues, domaines qu'elles qualifient d'une certaine manière. Par exemple, les publicités pour produits italiens nous ont habitués à percevoir et à penser l'Italie à travers un nombre limité d'images-types (pâtes, gondoles, tour de Pise, ...), chacune de ces images mettant en profil d'une certaine manière l'italianité: à travers sa cuisine, ses monuments, etc. Progressivement au sein des relations partie-tout typiques de la métonymie, se constituent des liaisons stables: les mêmes parties renvoient au même tout qui se signifie par les mêmes parties. D'autre part, certaines images deviennent de véritables matrices symboliques pour d'autres. Par exemple, dans la publicité et les magazines, certaines images fortes, constamment reprises, structurent métaphoriquement la représentation de la femme moderne: la femme-Aphrodite, l'athlète médaillée aux Jeux Olympiques, la ménagère avisée, la femme cadre d'entreprise, ...

L'encodage est à la base de la formation des codes. La publicité est évidemment un lieu privilégié pour la mise en évidence de ce processus. Mais on peut également l'observer dans la presse d'information. Soit un événement E; cet événement, en fait, est constitué 
d'un ensemble indéterminé de sous-événements interreliés $\left(\mathrm{e}_{1}, \mathrm{e}_{2}\right.$, $\left.e_{3}, \ldots\right)$. La survenue de cet événement engage les media à nous en informer, c'est-à-dire à nous livrer quelques représentations (verbales, photographiques, cinématographiques) de quelques sous-événements. Il est évidemment impossible de rendre compte de l'enchaînement complet de tous les événements. De plus, pour faciliter la compréhension, les sous-événements retenus le seront souvent en raison des analogies qu'ils permettent de construire avec d'autres événements (réels ou imaginaires) déjà inscrits dans la mémoire du public. Par exemple, $\mathrm{e}_{1}$ (des réfugiés, des belligérants armés, une poignée de mains, etc., etc.) sera choisi parce que son contenu et la représentation que l'on peut en construire par le verbe ou par l'image permet de rapprocher $\mathrm{E}$ de quelques prototypes d'événements (la guerre, la réconciliation, etc., etc.). Ainsi l'événement $\mathrm{E}$ sera-t-il connu; métonymiquement (c'est-à-dire très partiellement, à travers certaines de ses parties) et métaphoriquement (c'est-à-dire par analogie).

Il est inévitable qu'il en soit ainsi mais le processus peut être plus ou moins accentué. L'encodage consiste précisément à jouer de la mise en forme métonymico-métaphorique pour ramener le nouveau au connu. L'information, au fond, a le choix entre deux démarches opposées, entre lesquelles, bien sûr, existent des démarches intermédiaires. Dans la première, il s'agira de multiplier les vues partielles et les comparaisons, c'est-à-dire de donner de l'événement plusieurs aspects et de chercher les analogies les plus pertinentes. L'autre démarche -la démarche d'encodage- consiste à ne retenir de chaque événement que celui ou ceux de ses aspects qui permettent de l'assimiler le plus immédiatement à une longue série d'événements familiers déjà assimilés les uns aux autres. Dans le premier cas, l'événement apparaît complexe et reste extérieur aux prises du sujet dont la raison, de ce fait, est mobilisée. Dans le cas de l'encodage, au contraire, il y a enfermement de l'événement dans quelques schèmes typiques ou images emblématiques offerts à la reconnaissance immédiate sans autre effort de décryptage.

Étant donné la priorité accordée généralement au spectaculaire, la pratique d'encodage est dominante dans la presse contemporaine. Il ne faut pas que le sens des événements suscite trop d'interrogations; il faut plutôt qu'il frappe par son évidence car c'est la participation qui est sollicitée, non la réflexion. L'encodage implique la répétition du même et celle-ci implique du côté du sujet, une activité simple de reconnaissance passive qui en fait un simple récepteur-décodeur. 
L'encodage n'est pas coextensif à la communication -il n'en est qu'un des aspects- mais il est indispensable à la réalisation du modèle du code, celui dont nous avons vu l'importance pour le type de rapport social prédominant dans notre culture.

\section{La communication-stratégie}

Centrée sur l'usage des signes dans la communication plutôt que sur les questions relatives au sens ou à la transmission d'information, la pensée pragmatique a profondément transformé notre représentation de la communication, substituant à la métaphore du conduit et à l'image du télégraphe une nouvelle métaphore : la communication est action. L'ouvrage inaugural de Austin ("How to do things with words" traduit en français: "Quand dire, c'est faire") indique clairement le changement de point de vue. Communiquer, c'est "faire" quelque chose, au même titre que manger, marcher, jouer, etc. En effet, une énonciation verbale peut être caractérisée comme une affirmation, ou une promesse, ou un ordre, une interrogation, une menace, etc., toutes choses qui appartiennent à la représentation qu'on se fait du domaine de l'agir. Ce qui devient saillant dans cette manière particulière de mettre en perspective les choses de la communication, c'est la modalité spécifique de l'agir dans ce domaine: perçu comme action, le discour -comme du reste, toute forme de communication-apparaît surtout comme mise en forme de la relation interindividuelle, c'est-àdire: attribution de rôles, de positions, de places, etc. "En accomplissant un acte illocutionnaire, écrit Récanati, le locuteur s'assigne un certain rôle et assigne à l'auditeur un rôle complémentaire: en donnant un ordre, le locuteur exprime sa volonté que l'auditeur suive une conduite donnée, et se pose comme ayant l'autorité qu'il faut pour que l'auditeur soit obligé de suivre la conduite en question simplement parce que c'est la volonté du locuteur'l.

Le point de vue pragmatique a profondément modifié la recherche en communication. Il a détourné le regard des chercheurs des questions relatives aux codes, à l'information, à la signification pour le recentrer sur les différents aspects du rapport qui s'établit nécessairement, dans toute communication, entre un (des) destinateur(s) et un (des) destinataire(s). Il s'en faut cependant que les

1 François RÉCANATI, Les énoncés performatifs, Paris, Éd. de Minuit, 1981, p. 19. 
pratiques communicationnelles corrélatives au modèle du code s'en soient trouvées transformées. C'est que, dans une certaine mesure et paradoxalement, le modèle du code est sorti renforcé de sa confrontation avec la perspective pragmatique, du moins dans de nombreux secteurs de la communication. Il y a eu, au niveau de la représentation sociale de la communication, une interaction entre la vision télégraphique et la vision de la communication comme action qui a finalement abouti à donner à la première plus de force et de légitimité qu'elle n'en avait déjà. Tout vient de la manière dont nous concevons généralement l'action. La notion d'action est souvent à celle de force et de pouvoir. Dans la perspective de Lakoff, on dira que la notion d'action est métaphoriquement conçue en termes de force et de pouvoir. Que l'on considère par exemple le début de la définition du Petit Larousse: "Manifestation concrète de la volonté de quelqu'un, d'un groupe: son action a été déterminante; l'action du gouvernement. Manière dont agit un corps, une force...".

On voit les implications de cette assimilation métaphorique: la centration sur la composante action de la communication conduit inévitablement à se représenter schématiquement celle-ci comme l'exercice d'un certain pouvoir sur fond d'un rapport de force. Les meilleurs auteurs ont accrédité cette représentation. L'insistance de Récanati, dans le passage cité plus haut, sur l'exemple de l'ordre comme acte de discours -et sur la "volonté du locuteur", qui constitue un trait saillant de ce type d'acte- est un indice de la tendance à assimiler communication et pouvoir. Du reste, l'ordre -traduit théoriquement dans les notions de "directif" ou "prescriptif"- apparaît souvent comme un prototype d'acte de discours dans les textes de présentation de la pragmatique.

Un linguiste comme Ducrot a très explicitement rapproché les notions d'énonciation -au sens le plus général du terme- et de pouvoir:

"Troisième point que je veux souligner, dans la représentation de l'énonciation par l'énoncé: l'énonciation est caractérisée comme ayant certains pouvoirs. C'est de cette façon, en tout cas, que je comprends la théorie des actes illocutionnaires d'Austin. Dire qu'un énoncé est un ordre, une interrogation, une affirmation, une promesse, une menace, etc., c'est dire qu'il attribue certains effets à son énonciation, qu'il la présente notamment comme créatrice de droits et de devoirs. Comprendre, par exemple, l'énoncé de Viens comme un 
ordre, c'est supposer qu'il attribue à son énonciation le pouvoir d'obliger à venir quelqu'un qui, avant elle, n'avait pas cette obligation"1.

De nouveau, dans ce texte, l'ordre est implicitement pris comme prototype et l'exercice du pouvoir qui le caractérise est tout simplement étendu à toutes les catégories d'actes de discours.

Suivant la même inspiration, un philosophe comme Lyotard en arrive à décrire tout le domaine de la communication comme un vaste jeu à dominante agonistique:

"Et d'autre part la théorie de l'information dans sa version cybernétique triviale laisse de côté un aspect décisif, déjà souligné, l'aspect agonistique. Les atomes sont placés à des carrefours de relations pragmatiques, mais ils sont aussi déplacés par les messages qui les traversent, dans un mouvement perpétuel. Chaque partenaire de langage subit lors des "coups" qui le concernent un "déplacement", une altération, de quelque sorte qu'ils soient, et cela non seulement en qualité de destinataire et de référent, mais aussi comme destinateur. Ces "coups" ne peuvent pas manquer de susciter des "contre-coups"; or, tout le monde sait d'expérience que ces derniers ne sont pas "bons" s'ils sont seulement réactionnels. Car ils ne sont alors que des effets programmés dans la stratégie de l'adversaire, ils accomplissent celle-ci et vont donc à rebours d'une modification du rapport des forces respectives...

Ce qu'il faut pour comprendre de cette manière les rapports sociaux, à quelque échelle qu'on les prenne, ce n'est pas seulement une théorie de la communication, mais une théorie des jeux, qui inclut l'agonistique dans ses présupposés"2.

"Déplacements", "coups", "contre-coups", "stratégie", "rapports des forces", "jeux", tous ces termes superposent à l'idée de force les traits de deux grandes familles d'images: celle du combat et celle du jeu. C'est du reste le jeu d'échec, véritable projection-simulation du combat guerrier dans le domaine du jeu, qui semble bien avoir déterminé le choix du vocabulaire utilisé.

Ainsi, dans le contexte socio-culturel que nous connaissons, la métaphore générique de la communication-action a conduit la pensée -à travers divers circuits associatifs- à l'image spécifique du

1 Oswald DuCrot et Danièle BourCier, Les mots du discours, Paris, Éd. de Minuit, 1980, p. 36-37.

2 Jean-François LyOTARD, La condition post-moderne, Paris, Éd. de Minuit, 1979. 
communicateur-stratège exerçant un certain pouvoir, ou prenant position dans un jeu de rapports de force.

Or, cette représentation, c'est là qu'on voulait en venir, n'est pas contraire au modèle du code. Elle peut en effet se combiner avec lui, et cela dans la mesure même où la réalisation de ce dernier implique déjà, nous l'avons vu, l'exercice d'un certain pouvoir, la recherche d'une certaine influence par transformation du (des) destinataire(s) en récepteur(s)-décodeur(s) d'informations. La réalisation du modèle du code est bien affaire de stratégie; stratégie dans le choix des images qui frappent, séduisent, captent l'attention, stratégie dans les montages métonymico-métaphoriques par lesquels se réalise l'encodage des significations.

La métaphore de la communication-action ne fait qu'ajouter à l'image de la transmission à travers un conduit l'idée d'une force nécessaire pour que la transmission s'effectue. Superposables, les deux images se combinent et conjuguent leurs effets pour construire de la communication une représentation théorique ne retenant que ce qu'il y a en elle d'action persuasive: communiquer, c'est faire passer un message, l'imprimer dans la conscience du destinataire. Toute une conception des pratiques et métiers de la communication en dérive et s'en trouve justifiée. Les professionnnels de la communication -ou du moins une bonne partie d'entre eux- réfléchissent leur action en des termes empruntés au domaine de la stratégie de combat (public-cible, objectifs à atteindre, image-choc, ...) ce qui, d'une certaine manière, les contraint à développer toujours plus de technicité dans la persuasion.

Insistons- $y$, la représentation de la communication-action, avec tout ce qu'elle implique, n'est pas fausse. La communication est toujours, plus ou moins, action, combat, pouvoir, stratégie, persuasion; mais justement, elle est cela plus ou moins. Une représentation métaphorique, avons-nous vu, n'est pas forcément erronée. La métaphorisation est même sans doute nécessaire à la découverte de certains aspects des phénomènes que l'on tente de conceptualiser. L'ennui est qu'en mettant en lumière certains profils des choses, elle en tient d'autres dans l'obscurité et que, par l'effet des représentations sur les croyances et les conduites, elle engendre une contrainte en faveur de sa réalisation, tandis qu'elle joue un rôle inhibiteur à l'égard d'autres "réalités" possibles. La métaphorisation, d'une certaine manière, est une espèce d'exagération. 
Dès le moment où la plupart des gens s'entendent à penser que la communication est action stratégique, ils vont, afin de rendre leur conduite cohérente avec le modèle, exagérer en elle ce qui relève effectivement de l'action stratégique et inhiber ce qui s'y oppose, confirmant ainsi le modèle, lequel en retour, avalise les conduites, en boule de neige.

Mais une représentation ne peut jamais être que dominante. Un processus aussi complexe que la communication, aux aspects multiples, ne peut se laisser entièrement cerner par une représentation. Il existe toujours, conjointement à une représentation dominante, un ensemble indéterminé d'autres représentations, plus ou moins inhibées, relativement marginales par rapport à la représentation dominante, mais qui peuvent s'amplifier, devenir dominante à leur tour, catalyser de nouvelles conduites et une nouvelle réalité sociale.

Un rapprochement -une métaphore?- semble possible, ici, avec certains aspects des processus irréversibles décrits par le physicien Prigogine. Dans un système physico-chimique, explique Prigogine, il existe toujours, localement, des phénomènes déviants (par exemple, un petit mouvement de convection dans de l'eau chauffée où la chaleur se propage encore de manière dominante, par diffusion; l'apparition de petites gouttelettes dans un gaz,...) généralement inhibés, mais qui pensent, dans certaines conditions, au-delà d'un certain seuil, s'amplifier et générer une nouvelle organisation (les tourbillons de Bénard qui caractérisent l'eau qui bout, le changement d'état d'un gaz en liquide ...). Il en serait de même, pense Prigogine dans les systèmes biologiques et sociaux ${ }^{1}$. Et de fait, il semble bien qu'il en soit ainsi dans la dynamique des représentations sociales. Dans un système social dont la réalité dépend des représentations partagées par ses membres, il existe toujours de nombreuses représentations déviantes, marginales et vouées à l'insignifiance. Dans certaines conditions, suite à quelques ruptures d'équilibre, une représentation marginale et dysfonctionnelle par rapport au fonctionnement social global peut passer un seuil, s'amplifier par un processus autoaccéléré (boule de neige) où conduites et représentations s'entraînent l'une l'autre, et transformer le fonctionnement d'ensemble.

Se pourrait-il que le domaine de la communication connaisse une telle transformation? Que l'image de la représentation comme

1 Cf. Ilya Prigogine et Isabelle StEngers, La nouvelle alliance, Paris, Gallimard, 1979, notamment chapitre VI. 
transmission, codage, stratégie soit déstabilisée et qu'une nouvelle représentation passe un seuil et s'impose à son tour, générant de nouvelles conduites et transformant du coup la réalité sociale?

\section{La métaphore de l'ordinateur et le modèle cognitif}

De fait, la communication semble être l'objet d'une nouvelle mise en profil dégageant des aspects jusqu'ici largement ignorés -les aspects cognitifs de la communication- et, ce qui est plus intéressant, apparemment antagonistes aux aspects jusqu'ici entrevu (code et stratégie).

Ces aspects ont commencé à se signaler à l'attention des chercheurs avec des problèmes du genre de celui-ci: qu'est-ce qui, dans une conversation entre $\mathrm{A}$ et $\mathrm{B}$, permet à $\mathrm{B}$ de comprendre que $\mathrm{A}$ veut disposer de son temps pour étudier lorsqu'à l'invitation de $\mathrm{B}$ d'aller au cinéma il répond: "Cela durerait trop longtemps". La réponse à un tel problème conduit à l'idée que la compréhension d'un énoncé nécessite toujours une certaine activité d'inférence. L'imagerie liée au monde des ordinateurs, de l'intelligence artificielle, de la computation, a certainement aidé à donner à de tels aspects le relief nécessaire pour que, peu à peu, ils soient considérés comme centraux. L'intérêt pour la computation a renouvelé et avivé l'intérêt pour la cognition et celleci est devenue si saillante qu'aucune conduite, aucune activité humaine, ne peut se décrire et s'expliquer sans qu'on lui fasse sa part. Un seuil semble être passé avec l'idée que tout compte fait, la cognition est plus essentielle à la communication que l'activité de codage/décodage où tout autre aspect; cette idée a déjà fait l'objet d'une importante élaboration théorique de la part d'auteurs tels que Sperber et Wilson ${ }^{1}$.

Au-delà de ce seuil, c'est toute la problématique de la communication qui se restructure autour de nouvelles attitudes de recherche, hypothèses, propositions. On peut d'ores et déjà relever quelques grandes tendances caractéristiques de cette restructuration. Il est de plus en plus évident que la langue -comme du reste tout code- ne peut être considérée comme une entité autonome que l'on peut étudier indépendamment de l'activité intellectuelle et sociale des êtres humains. Cela, que la pragmatique d'inspiration austinienne avait déjà

1 Cf. Dan Sperber et Deirdre Wilson, op. cit. 
affirmé, trouve maintenant une traduction cognitive dans les affirmations des linguistes de la sémantique cognitive.

"Après une génération de chercheurs qui admettaient implicitement que le langage pourrait être décrit en ses propres termes, il est devenu plus intéressant de se demander quelle part de la structure du langage est déterminée parce que les hommes ont un corps avec des sens pour la perception, une mémoire, des capacités d'enregistrement et des limitations, par le fait que les hommes doivent essayer de donner un sens au monde avec des ressources limitées et par le fait que les hommes vivent en groupes sociaux et doivent essayer de communiquer entre eux"1.

Examinés dans une perspective cognitive, les différents systèmes de signes (langage parlé, langage écrit, image fixe ou animée, représentations graphiques ou infographiques, ...) apparaissent comme autant de technologies de l'intelligence aux implications multiples ${ }^{2}$.

La perspective cognitive oblige également à concevoir autrement l'échange communicationnel. Dans la perspective pragmatique classique, l'énonciation apparaît encore comme une affaire individuelle: elle est action d'un individu (le locuteur ou plus généralement l'énonciateur) sur un autre individu. Mais dès lors qu'il devient évident que le destinataire d'un message doit, ne fut-ce que pour comprendre celui-ci, raisonner, inférer, construire des "modèles mentaux" à partir des quelques indices que lui fournit le message, il devient évident aussi que l'énonciation constitue une activité collective ou intersubjective, relevant autant du destinataire que du destinateur. Des notions comme celles de co-énonciation ou co-construction (du sens) proposées par certains auteurs, essayent de rendre compte de cette nécessaire collaboration dans l'interlocution.

"La co-énonciation est l'ensemble des rapports complexes que tissent les deux interlocuteurs au moyen du langage. La représentation n'est ni neutre, ni objective, c'est un processus médiatisé par les sujets de construction et de re-construction de la signification; elle est indissociable des deux protagonistes de

1 LAKOFF cité par D. GEERAERTs, "Grammaire cognitive et sémantique lexicale", in Communication, $\mathrm{n}^{\circ}$ 53, op. cit., p. 29-30.

2 Cf. à ce sujet Pierre LÉVY, op, cit. 
l'échange verbal ainsi que des représentations, images et visées de l'un par l'autre (...)"'.

De telles notions, on s'en rend compte, relativisent sérieusement celle du récepteur.

On ne peut ici détailler davantage ces grandes tendances de l'approche cognitive. L'important, à ce point, est de montrer la réorientation et le redéploiement qu'elles peuvent imprimer aux pratiques concrètes de communication.

L'attention à la dimension cognitive de la communication implique tout d'abord un changement de norme, un renversement dans la manière de concevoir ce que peut être une bonne communication ou une communication réussie. C'est, nous l'avons vu, la définition théorique qui donne les critères d'appréciation de la pratique: une bonne pratique est celle qui réalise la définition. Dans le cadre de la communication-action-stratégie, est considérée comme communication réussie celle qui atteint son but -faire passer le message- quel que soit le coût cognitif (annihilation des capacités de raisonnement sous l'effet des images-chocs et de l'évidence des codes), lequel n'est même pas aperçu. Dans le cadre de la communication-cognition, le critère d'appréciation sera nécessairement cognitif. Comment, dans ce cadre, apprécier une communication, définir ce qu'est une bonne communication ou une communication réussie autrement qu'en prenant en compte les caractéristiques des opérations cognitives (nature, qualité, niveau, complexité) sollicitées? Quel autre but donner à la communication que de réaliser l'optimum de coopération cognitive dans l'échange?

Dans la mesure où les critères d'appréciation emportent les pratiques, on peut alors s'attendre à des changements à différents niveaux. Au niveau de l'approche psychosociologique de la relation: on n'aborde pas l'interlocuteur (ou le public dans le cas des media) de la même façon selon qu'on veut agir sur lui, ou selon que l'on veut interagir avec lui sur le plan de la représentation cognitive du monde. Aux niveaux sémiologique et technologique: dès lors que ce qui est visé, c'est la cognition, les systèmes de signes et les supports techniques permettant la meilleure activation des facultés cognitives dans l'interaction seront nécessairement favorisés. Cela implique sans doute une redistribution et une modification des principales composantes

1 C. FuChS, cité par A. Trognon in Rodolphe GHIGLIONE, L'homme communiquant, Paris, Armand Colin, 1986, p. 28. 
(verbale, iconique, écrite, ...) intervenant dans les messages médiatiques. Par exemple, pour ne prendre que la composante iconique, on peut s'attendre à ce que le type d'image figurative auquel nous a habitués la communication publicitaire -lequel favorise essentiellement l'appréhension mimétique des choses et des êtres- se fasse moins dominante et qu'émergent et se développent des formes iconiques réclamant davantage de distanciation et s'adressant à des formes plus complexes d'élaboration cognitive: par exemple les schémas, diagrammes, cartes, réseaux, etc. que permettent les méthodes de représentation graphique et l'informatique.

Il en est de même sur le plan technologique. Dans la perspective cognitive, il est vraisemblable que se développent, parmi les nouvelles technologies de communication, celles qui favorisent l'interaction cognitive. Il est d'ores et déjà remarquable que les hypermedia semblent intéresser davantage les chercheurs concernés pas la cognition et la communication des savoirs que les stratèges de la persuasion.

Avec les théories cognitives de la communication, c'est tout un style nouveau d'interaction sociale qui se laisse entrevoir, peut-être quelque chose que l'on pourrait nommer, selon la belle et stimulante expression proposée par E. Morin, une "démocratie cognitive".

\section{Conclusion}

Il n'y a pas de solution de continuité entre la culture, les représentations théoriques, les conduites et les rapports sociaux. Tous ces éléments s'ajustent les uns dans les autres. Loin d'être de simples reflets de la réalité, les représentations théoriques agissent comme des formes catalysant les réactions capables de leur donner du relief. Ainsi des théories de la communication. Il faut y insister encore, aucune de ces théories n'est fausse. Simplement, chacune d'elles joue comme un filtre, mettant certains aspects des choses en relief et laissant d'autres dans l'ombre. Souvent, ce sont des métaphores qui font office de filtres. Les structures conceptuelles -qui sont des structures cognitives- se moulent dans les images -souvent d'origine technologique- qui hantent l'imaginaire social. Et les comportements suivent, collaborant tacitement à la réalisation de l'image dominante; du moins à un certain degré de réalisation, car la communication reste dans tous les cas un phénomène complexe où coexistent toujours plusieurs composantes. L'hypertrophie de l'une d'entre elles n'entraîne 
pas l'anéantissement des autres. Quels que soient les modèles et pratiques dominants, il y a toujours un minimum de codage, d'action, de stratégie, d'activité cognitive, etc. dans la communication. Peut-être est-ce du reste la répression d'une composante -c'est-à-dire sa réduction au minimum- qui occasionne son réveil et permet que s'amorce un mouvement de transformation en sa faveur.

Pendant des années, des images de conduits, de tuyaux, de transport, d'action, d'impact, ont inspiré, justifié, banalisé une vision des choses, éthique et pratique, entrainnant la réalité sociale à être prioritairement un vaste champ de bataille, un vaste système de rapports de force.

Dans ce champ, peu de place pour la cognition, si bien qu'on peut comprendre les nouvelles images -d'ordinateur, de traitement d'information, de computation, etc.- comme l'indice du retour de cette composante oubliée et l'amorce d'une transformation entraînant la réalité sociale à devenir prioritairement -ou au moins un peu plus- un vaste réseau d'esprits interconnectés.

Telles étaient, pour l'essentiel, les vues exprimées dans cet article. Il me reste à souhaiter qu'elles aient tendance à se réaliser. 